Wire-bonder-assisted integration of non-bondable SMA wires into MEMS substrates

This article has been downloaded from IOPscience. Please scroll down to see the full text article.

2012 J. Micromech. Microeng. 22055025

(http://iopscience.iop.org/0960-1317/22/5/055025)

View the table of contents for this issue, or go to the journal homepage for more

Download details:

IP Address: 130.237.43.49

The article was downloaded on 19/04/2012 at 12:21

Please note that terms and conditions apply. 


\title{
Wire-bonder-assisted integration of non-bondable SMA wires into MEMS substrates
}

\author{
A C Fischer, H Gradin, S Schröder, S Braun ${ }^{1}$, G Stemme, \\ $W$ van der Wijngaart and F Niklaus
}

\author{
Microsystem Technology Laboratory, School of Electrical Engineering, KTH Royal Institute of \\ Technology, Osquldas väg 10, 10044 Stockholm, Sweden \\ E-mail: andreas.fischer@ee.kth.se
}

Received 2 December 2011, in final form 28 February 2012

Published 19 April 2012

Online at stacks.iop.org/JMM/22/055025

\begin{abstract}
This paper reports on a novel technique for the integration of NiTi shape memory alloy wires and other non-bondable wire materials into silicon-based microelectromechanical system structures using a standard wire-bonding tool. The efficient placement and alignment functions of the wire-bonding tool are used to mechanically attach the wire to deep-etched silicon anchoring and clamping structures. This approach enables a reliable and accurate integration of wire materials that cannot be wire bonded by traditional means.
\end{abstract}

(Some figures may appear in colour only in the online journal)

\section{Introduction}

Wire bonding is a highly mature, cost-efficient and broadly available back-end process for electrical interconnects and the most commonly used interconnection method in integrated circuit packaging [1-3]. These features make wire bonding a versatile tool even for the integration of wires for applications beyond electrical chip interconnects. In recent research activities, unconventional wire-bonding approaches have been developed to implement a variety of innovative applications such as microcoils for energy harvesters [4] and MR imaging/spectroscopy [5], transformers [6], on-chip antennas [7], through silicon vias [8-10] and hermetic liquid encapsulation [11].

The wire-bonding process is in principle a welding technique in which a metal microwire is attached to a metal bond pad. The energy input for this metal/metalwelding process is a combination of force, temperature and/or ultrasonics. Figure 1 illustrates the standard thermosonic ball/stitch bond process of gold wire to gold or aluminum pads. As shown in figure 1(a), the gold wire is fed through a ceramic bond capillary and an electrical discharge (flame-off) then melts the wire and forms a gold sphere, the free air ball

\footnotetext{
${ }^{1}$ Present address: Robert Bosch GmbH, Reutlingen, Germany.
}

(FAB), at the end of the wire. Thereafter, the FAB is pulled up to the tip of the capillary and the tool moves laterally to a position above the desired pad on the substrate, as depicted in figure $1(b)$. The tool presses the FAB with a defined force against the pad. The elevated temperature from the substrate is placed on a heated work piece holder and a simultaneous input of ultrasonic energy forms the weld between the metal ball and the metal pad (figure 1(c)). Then, the tool moves toward the second bond pad where the stitch bond is performed (figure 1(d)). As shown in figure 1(e), the wire is compressed between one side of the capillary tip and the pad. Again, the combination of force, ultrasonics and temperature creates the weld between the wire and the pad. Finally, the tool moves up to a certain height (figure $1(f)$ ), where the wire is torn off and the process can start over again (figure $1(g)$ ).

All types of wire-bonding processes based on welding are limited to certain material combinations and can be sensitive to imperfections and contamination of the bond surfaces $[1,2]$. Commercially relevant wire-pad material combinations are $\mathrm{Au}-\mathrm{Au}, \mathrm{Au}-\mathrm{Cu}, \mathrm{Au}-\mathrm{Pd}, \mathrm{Al}-\mathrm{Au}$ and $\mathrm{Al}-\mathrm{Ni}$. Strongly emerging combinations are $\mathrm{Cu}-\mathrm{Al}$ and $\mathrm{Cu}-\mathrm{Cu}$ due to a more attractive commodity price of copper as compared to gold [2]. Other more exotic wire-pad combinations such as $\mathrm{Pd}-\mathrm{Al}$ [12, 13], Pt-Pt [14], Ni-SiC [15] and Ag-SiC [16] have been reported as well. The hardness of the used wire material has 
(a)

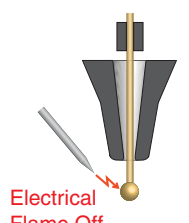

Flame Off (b)

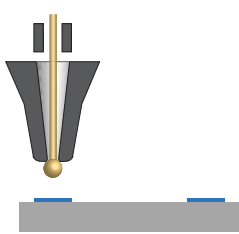

$\uparrow \uparrow$ Temperature $\uparrow \uparrow$ $(c)$

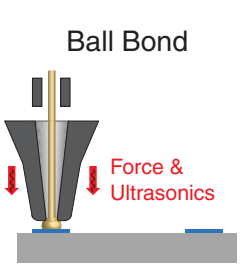

(d)

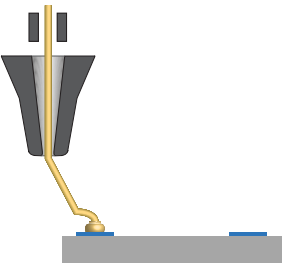

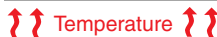

$(e)$

$(f)$

(g)

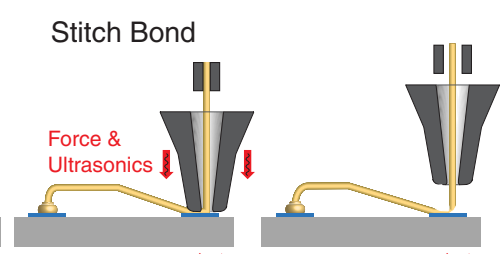

Figure 1. The process flow of the standard thermosonic ball/stitch bonding of gold wire. A FAB is ball-bonded to a metal pad, and after generating a specific loop shape of the wire, it is stitch-bonded to the second bond pad.

Table 1. Vickers hardness of standard metals used for wire bonding such as gold, aluminum and copper. Platinum, palladium, nickel and silver wires are known to be bondable as well. Wire bonding has not been demonstrated for equiatomic NiTi SMAs due to the extreme hardness of the material class.

\begin{tabular}{ll}
\hline Material & Vickers hardness [MPa] \\
\hline $\mathrm{Au}$ & $245[18]$ \\
$\mathrm{Al}$ & $147[18]$ \\
$\mathrm{Cu}$ & $490[18]$ \\
$\mathrm{Pd}$ & $362-1030[18]$ \\
$\mathrm{Pt}$ & $392[18]$ \\
$\mathrm{Ni}$ & $981[18]$ \\
$\mathrm{Ag}$ & $245[18]$ \\
$\mathrm{NiTi}($ SMA) & $2000-2300[19]$ \\
\hline
\end{tabular}

an important impact on the bondability and the reliability of the bond. It is known that material failures, such as cratering, are more likely to occur for hard wire materials [2, 3]. Table 1 lists the Vickers hardness of selected materials. Wire bonding is typically based on welding soft wire materials to other soft or, in rare cases, to hard metal pad surfaces. It has also been shown to be possible to bond gold wires to silicon structures with the help of standard wire-bonding tools. In this case, the bond mechanism is not based on metal/metal welding but on a plastic deformation of a ball bond into a silicon hole, which results in a mechanical attachment [17]. This method however requires a wire with a low hardness in order to enable a plastic deformation of the wire without deforming or damaging the substrate.

Shape memory alloys (SMAs) are very attractive actuator materials for microelectromechanical system (MEMS) applications, especially when high forces and large displacements are desired. At the microscale, the work density of SMA materials is at least one order of magnitude higher than other actuation mechanisms, such as electrostatic, piezoelectric and magnetic actuation [20]. Traditionally, there are mainly two ways of integrating SMA materials into microsystems. Most common are sputter deposition or evaporation of thin SMA films directly onto the microstructure [20]. This method enables wafer-level processing. However, because of difficult deposition control, the process is limited with respect to the achievable layer thickness and reproducibility of transformation temperatures and strains [21]. The second approach for the integration of SMA wires into microsystems is to use bulk SMA materials. The MEMS structures and the SMA material are first fabricated separately and then combined on a device level by a pick and place approach [22]. Bulk SMA materials are commercially available in a wide thickness range at comparably low material cost, but integration on a per device level results in high assembly cost. Recently, wafer-level heterogeneous integration processes [23] for bulk SMA sheets using both adhesive [24] and Au-Si eutectic bonding [25] have been presented for the creation of actuators and microvalves. Also, wafer-level integration processes for SMA wires have been presented for the creation of high performance actuators, using both polymers [26] and $\mathrm{Ni}$ electroplating [27] for attaching the wires to the substrates. However, these wireintegration approaches suffer from a number of problems and disadvantages. First, a dedicated frame is needed to hold the SMA wires in place and the placement of the wires in the frame is performed by hand. Second, the frame with the wires needs to be manually aligned to a pre-processed silicon wafer under a microscope. Wire-bonding tools would be ideal to replace the manual alignment and attachment of the wires. However, direct wire bonding of NiTi SMA wires is not feasible using traditional ball/stitch techniques due to the excessive Vickers hardness of NiTi, which is one order of magnitude higher as compared to Au (see table 1).

This paper presents a method for the alignment, placement and mechanical attachment of SMA wires that are not wire bondable by traditional means [28]. The method enables the cost-efficient fabrication of high-performance actuators, such as microvalves and tunable RF and optical devices.

\section{Concept for wire fixation by anchoring and clamping}

In this paper, a generic method for the integration of nonbondable wire materials is presented. This approach is demonstrated with NiTi SMA wires, which are commercially available in a wide thickness range from 25 to $500 \mu \mathrm{m}$ [29]. The proposed method utilizes a conventional and unmodified wire-bonding tool. However, the attachment of the wire to the substrate is not realized by traditional means of wire bonding (i.e. metal/metal welding). Instead, the wire is attached and mechanically fixated and clamped by micromachined silicon structures.

Figure 2 illustrates the novel wire-integration scheme. The FAB acts as an anchor that is attached to a deep- and under-etched silicon structure using the wire-bonding tool. As illustrated in figure 2(a), the anchoring structure consists of two features, which are a landing zone and the actual tapered 
(a) Anchor

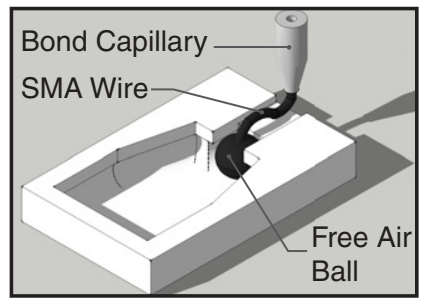

(b) Clamp

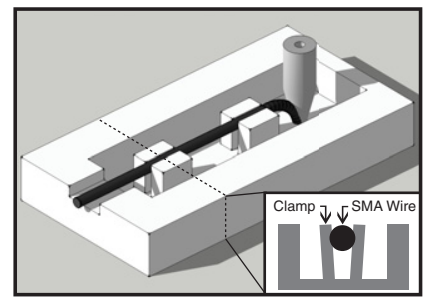

(c) Wire Integration

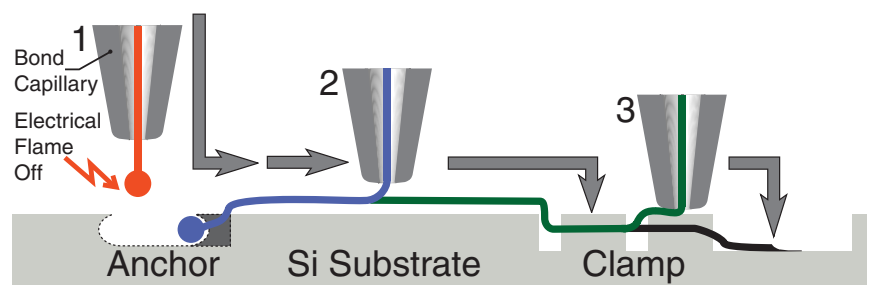

Figure 2. Illustration of the mechanical fixation structures used for anchoring and clamping of a wire. The wire is integrated with the help of a wire-bonding tool. The wire is anchored with the help of the FAB at one end and then fixated by a clamp structure at the other end.

fixation structure. The bond tool lowers the FAB above the landing zone until it touches the ground; then, it moves laterally toward the tapered fixation structure (figure 2(c), step 1). This fixation structure self-centers and fixates the FAB due to its inplane tapered and under-etched features. The tool then moves toward the second attachment position (figure 2(c), step 2), where the wire is fixated by a clamp structure, which also is a deep- and under-etched silicon structure. The wire-bonding tool pushes the wire with a defined force into the clamp without deforming the wire, as indicated in figure 2(c), step 3. Finally, the wire is truncated by applying a high force and ultrasonic energy. The attachment of the wire to the substrates with the help of the anchor and clamp structures is purely mechanical. The presented attachment technique is performed under roomtemperature conditions, which is essential in order not to trigger the SMA effect for applications using pre-strained SMA wires. The design and fabrication of the attachment structures is presented in the following section.

\section{Fabrication and integration process}

The presented concept for the integration of non-bondable wires is divided into a front- and a back-end process. The frontend process includes the fabrication of fixation structures by deep reactive ion etching of silicon. In the back-end process, the wire is integrated into the fixation structures utilizing a conventional wire bonder.

\subsection{Deep etching of the anchor and clamp structures}

The fabrication is based on $100 \mathrm{~mm}$ double-side polished silicon wafers and a Surface Technology Systems (STS) Multiplex Inductive Coupled Plasma (ICP) etch reactor is used for the deep etching of the fixation structures. The anchor structures are fabricated using the process flow depicted in
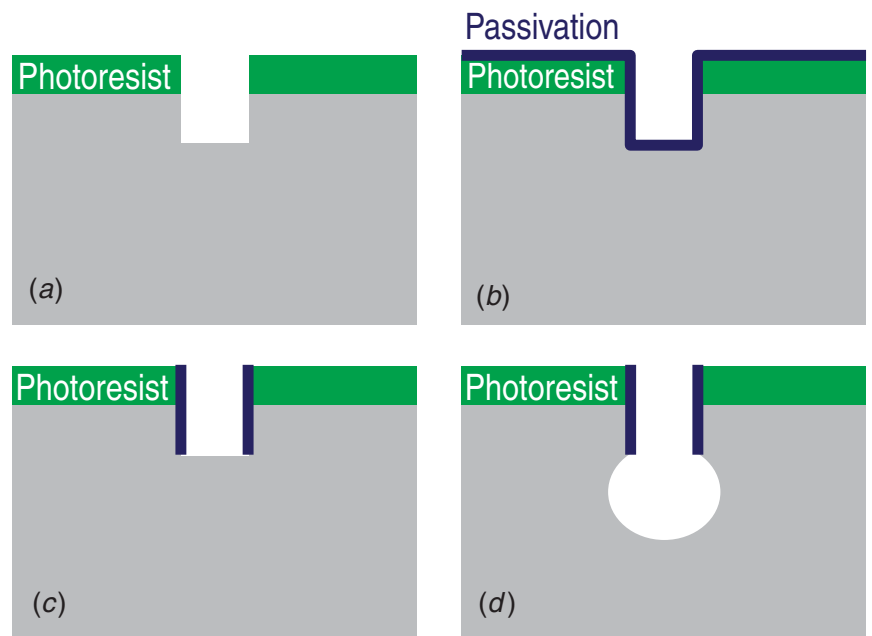

Figure 3. The process flow for the anchor structures. (a) An anisotropic etch is followed by $(b)$ a blank passivation, which is then removed $(c)$ at the horizontal surfaces. An isotropic etch $(d)$ creates the characteristic undercut for the anchor structures.
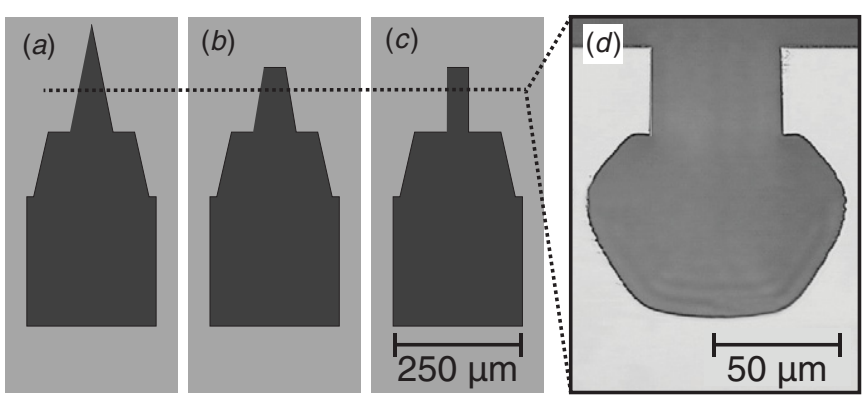

Figure 4. Three different anchor design variations are investigated. $(a),(b)$ A tapered surface opening of the anchor structure and (c) a rectangular design, which is matched to a specific wire diameter.

(d) Microscope image of the cross-section of an anchor structure.

figure 3. The process is similar to a single-crystal reactive and metallization (SCREAM) process [30], which enables etch profiles with buried undercuts. First, a vertical trench is etched by a standard anisotropic deep etch (figure 3(a)). A blank polymer deposition follows in order to protect the vertical sidewalls during the subsequent isotropic etching sequence (figure $3(b)$ ). The polymer on horizontal surfaces are removed by a physical sputter etch, as indicated in figure 3(c). A final isotropic etch sequence with tuned horizontal and vertical etch rates forms the desired undercut for the anchor structure, as illustrated in figure $3(d)$. The resulting total etch depth of the process sequence is approximately $100 \mu \mathrm{m}$. The detailed process parameters are listed in table 2 .

Three different design variations of the anchor structure are investigated in this work, as illustrated in figure 4. All three design variations have identical landing zones. The design variations depicted in figure $4(a)$ and $(b)$ allow variations of the wire and ball diameter due to their tapered profile. The rectangular design, depicted in figure $4(c)$, is designed for a single specific wire diameter of $37.5 \mu \mathrm{m}$. The microscope image in figure $4(d)$ depicts the cross-section of the final etch profile of the anchor structure. Differences in terms of the functionality of the structures are discussed below. 
Table 2. Main dry etch process parameters for each step of the fabrication of the anchor structures. A Surface Technology Systems (STS) Multiplex ICP is used in this work.

\begin{tabular}{|c|c|c|c|c|c|c|c|c|}
\hline $\begin{array}{l}\text { Process } \\
\text { step }\end{array}$ & $\begin{array}{l}\text { Process } \\
\text { sequence }\end{array}$ & $\begin{array}{l}\text { Time } \\
(\mathrm{sec})\end{array}$ & Cycles & $\begin{array}{l}\text { Platen } \\
\text { power (W) }\end{array}$ & $\begin{array}{l}\text { Coil } \\
\text { power (W) }\end{array}$ & Gases & $\begin{array}{l}\text { Flow } \\
(\mathrm{sccm})\end{array}$ & $\begin{array}{l}\text { Chamber } \\
\text { pressure [mbar] }\end{array}$ \\
\hline \multirow[t]{2}{*}{1 (figure $3(a))$} & Anisotropic: & & 44 & & & & & \\
\hline & -Etching & 7 & & 13 & 600 & $\mathrm{SF}_{6}, \mathrm{O}_{2}$ & 150,10 & 0.04 \\
\hline 2 (figure $3(b)$ ) & Passivation & 360 & 1 & 0 & 600 & $\mathrm{C}_{4} \mathrm{~F}_{8}$ & 100 & 0.027 \\
\hline
\end{tabular}

Table 3. Main dry etch process parameters for each step of the fabrication of the clamp structures.

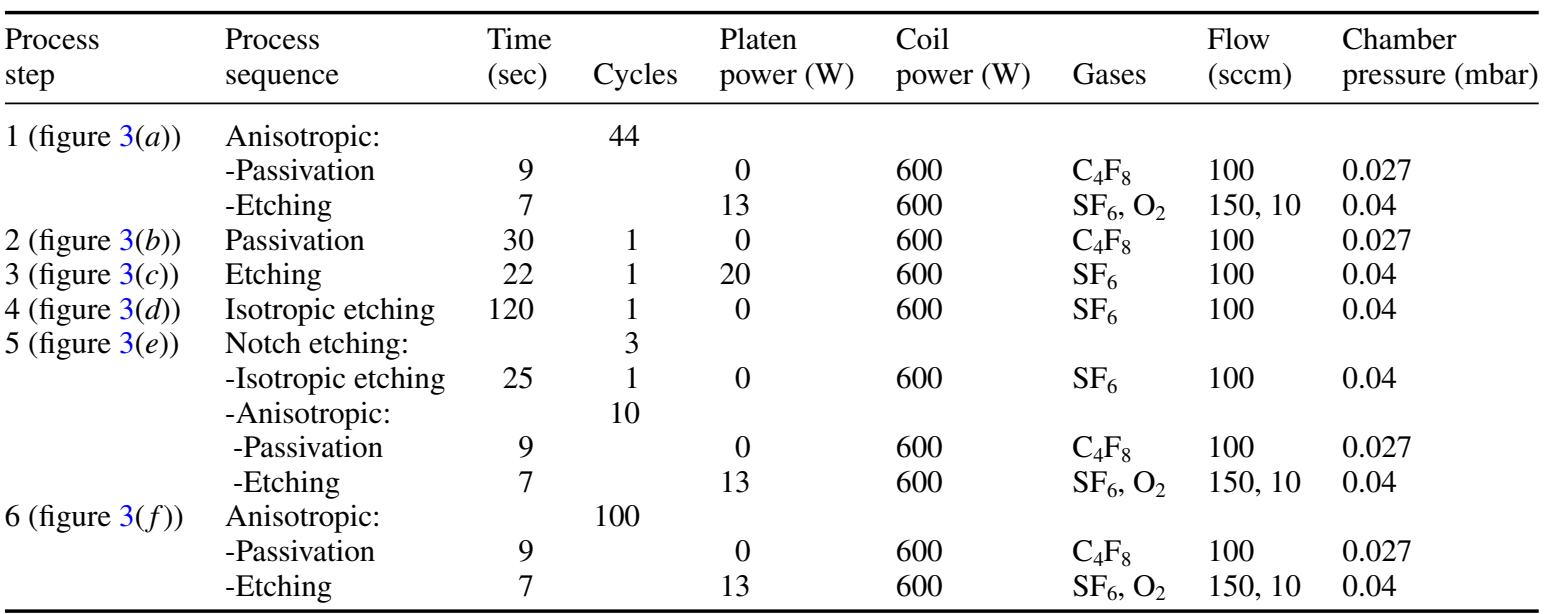
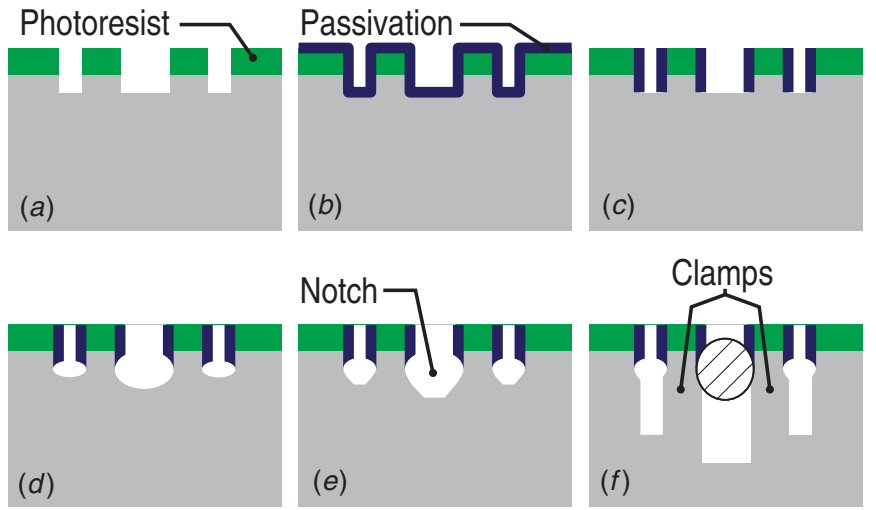

Figure 5. The process flow for the clamping structures. The resulting etch profile is achieved by a sequence of anisotropic, isotropic and passivation cycles. The notch is implemented to fixate the wire in a defined height in the clamp. As the beams of the clamping structure will bend slightly outward while the wire is integrated, it is required to provide sufficient space to the right and left of the clamp structure.

The clamping structures consist of two facing cantilevers with a notch, which is used to fixate the wire in a defined position and to prevent it from slipping out of the clamp. A similar etch process as for the fabrication of the anchor structures has been used but enhanced by additional process steps in order to create the notch. Figure 5 depicts the process flow for the fabrication of the clamping structures. A vertical trench is formed by an anisotropic deep etch (figure 5(a)); subsequently, a passivation layer is deposited, as illustrated in figure $5(b)$. A sputter etch removes the polymer on horizontal surfaces (figure 5(c)). The notch on both clamps is fabricated by a short isotropic etch step and a tapered deep etch, as depicted in figures $5(d)$ and $(e)$. The tapered etch for the notch consists of successive switching between anisotropic deep etching and isotropic etching. This creates a defined sidewall angle of approximately $20^{\circ}$. The notch is used to fixate the wire at a defined position in the clamp as illustrated in figure $5(f)$. An anisotropic deep etch completes the final cantilever geometry that provides a mechanical clamping of the wire and an adjacent lower plane for the wire truncation. The resulting total etch depth of the process sequence is approximately $200 \mu \mathrm{m}$. Table 3 summarizes the sequence of the used process steps as well as the process parameters.

In order to determine the clamping performance, a variation of clamping structures with different gap widths $(24,27,30 \mu \mathrm{m})$ was fabricated. Figure 6(a) shows the top view of a clamping structure, which consists of four pairs of silicon clamps that are arranged in a row. Each cantilever has a base area of $200 \mu \mathrm{m} \times 1000 \mu \mathrm{m}$ and a height of $200 \mu \mathrm{m}$. A cross-section of the clamping structure with a detailed view on the notch is shown in figure $6(b)$. The performance of the clamping structures is discussed below.

The anchor and clamping structures are fabricated in two separate process sequences, as discussed above. Each of the two process sequences uses an individual mask. The clamping structures require higher precision and a more elaborate etch profile; hence, the clamps are fabricated first. The lithography is performed with the photoresist SPR Megaposit ${ }^{\mathrm{TM}} 700-1.2$. The topography of the clamping structures does not allow a reliable coverage with resist that is applied by spin coating. 


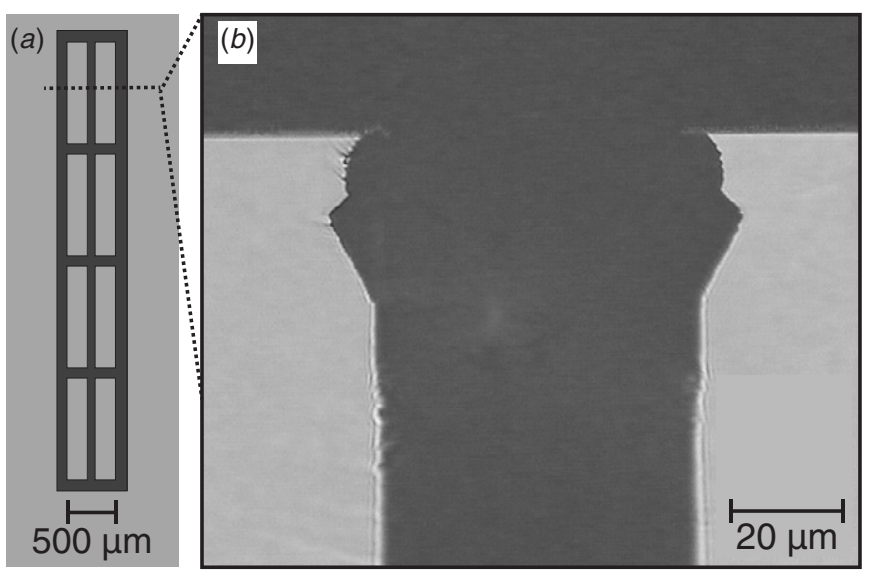

Figure 6. Detailed view on the etch profile of the upper part of the clamping structure. The notch in which the SMA wire is clamped is distinctly visible.

Therefore, the photoresist for the lithography of the anchor structures was applied by spray coating.

\subsection{Wire-integration process using wire-bonding techniques}

A conventional wire bonder (F \& K Delvotec 5410) was utilized for the mechanical attachment of the SMA wire. This tool is intended for thermosonic ball/stitch bonding of gold wires with diameters between 17.5 and $50 \mu \mathrm{m}$ and can be used in a manual mode. For the implementation of the integration process of the proof-of-concept, a manual tool was chosen in order to have full control over the individual process step. Fully automated tools are typically restricted to regular wire bonding, i.e. limited control and possibilities of customizing the bond process. This problem can be overcome by customized software solutions that allow one to define 3D arbitrary loop shapes [31], for example. The following section describes details of the formation of FABs on SMA wires as well as the wire-integration process in detail.

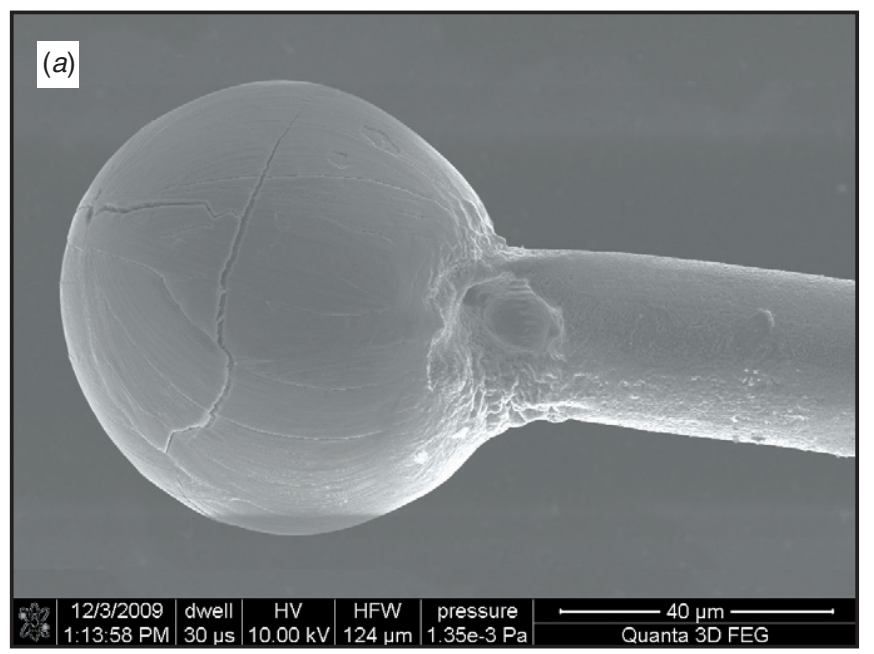

Experiments for a reliable FAB formation have been performed with the aforementioned wire-bonding tool, which is equipped with a Uthe 228-1 flame-off unit. The flameoff unit generates a high-voltage discharge, which melts the wire locally at the end of the bond wire and forms a metal sphere, the FAB, at the end of the wire. All experiments were performed with commercially available pre-strained SMA Flexinol wires with a diameter of $37.5 \mu \mathrm{m}$ and a transition temperature of $90{ }^{\circ} \mathrm{C}$. The flame-off experiments have been initially performed in air atmosphere and resulted in deformed FABs with brittle surfaces and weak ball/wire interfaces. Figure 7 $(a)$ depicts a SEM image of a typical FAB formed in an air atmosphere. The FAB has a colored pattern on its surface and the heat-affected zone of the wire has colored zones as well. Pull tests revealed an insufficient mechanical stability and very weak ball/wire interfaces. An energy-dispersive xray spectroscopy analysis on a cross-section of a FAB was performed. The analysis showed that the outer and brittle shell consists of strongly oxidized titanium. The remaining TiNi composition consists of nickel-rich phases that are formed towards the center of the FAB. This effect has been identified as a failure mechanism [32]. Other FAB experiments were performed in an inert atmosphere in order to avoid any oxidation reactions of the SMA [33]. Electrical flame-off experiments in an inert helium atmosphere led to mechanically stable FAB/wire interfaces. Figure 7(b) depicts a FAB, which is generated in an helium atmosphere. This FAB was formed with a voltage of $2.5 \mathrm{kV}$, a current of $42.6 \mathrm{~mA}$ and a firing time of $9 \mathrm{~ms}$. The diameter of the resulting FAB is $65 \mu \mathrm{m}$. Oxidation of titanium was avoided and the FAB has a smooth interface with a similar surface texture as the wire.

The integration of SMA wires according to figure 8 is performed in the manual bonding mode. A conventional bond capillary (UTS-68-CM-1/16XL, SPT Roth Ltd, Switzerland) with a hole size of $68 \mu \mathrm{m}$ was used for all experiments. First, a FAB is formed by an electrical flame-off in a helium atmosphere (figure 8(a)) and subsequently lowered toward the landing zone of the anchoring structure, as depicted in

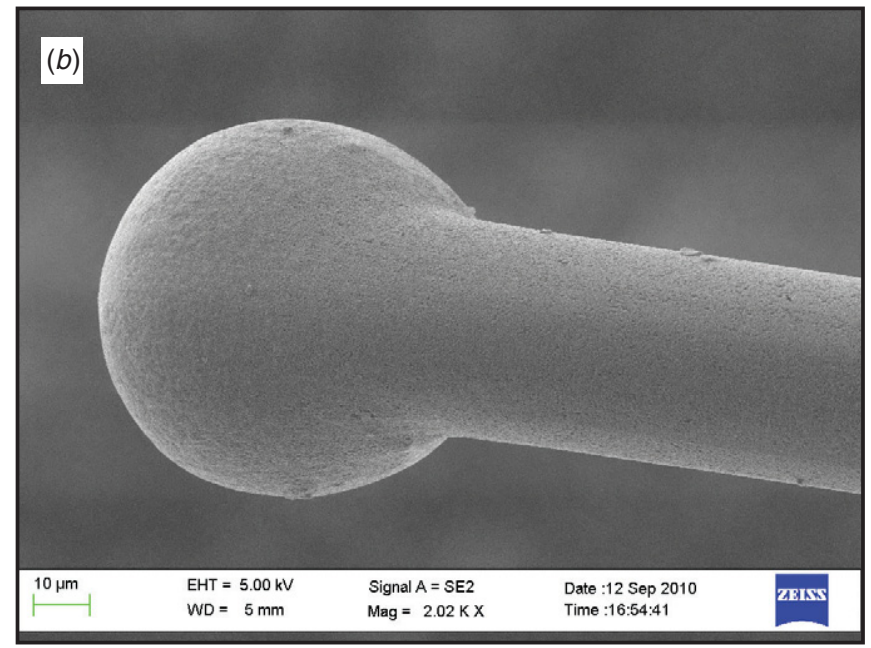

Figure 7. (a) SEM image of a typical FAB, which was formed in an air atmosphere. The interface between the ball and wire is crumpled and mechanically unstable. (b) SEM image of a FAB with a spherical shape and a smooth surface, which has been formed in a helium atmosphere. 

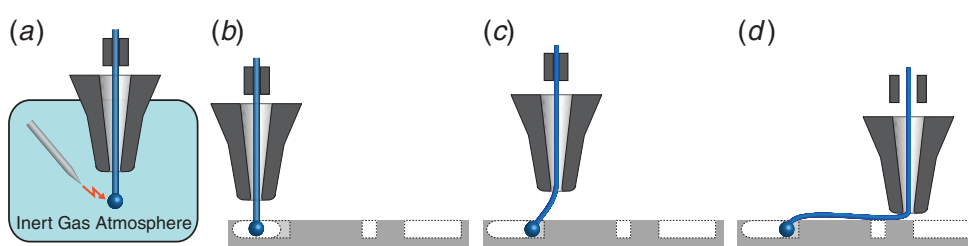

$(e)$

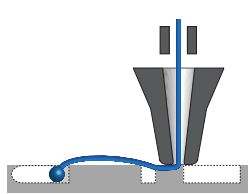

$(f)$

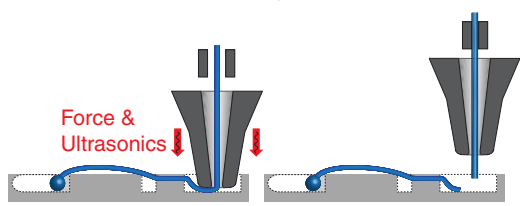

Figure 8. Detailed process flow for the integration of unconventional wire types. A FAB is formed and anchored in an under-etched silicon structure. The wire is then fed above a clamp structure. By applying a force on the wire, it is pressed into the clamp and thereby fixated. As a final step, the wire is truncated by applying force and ultrasonics.
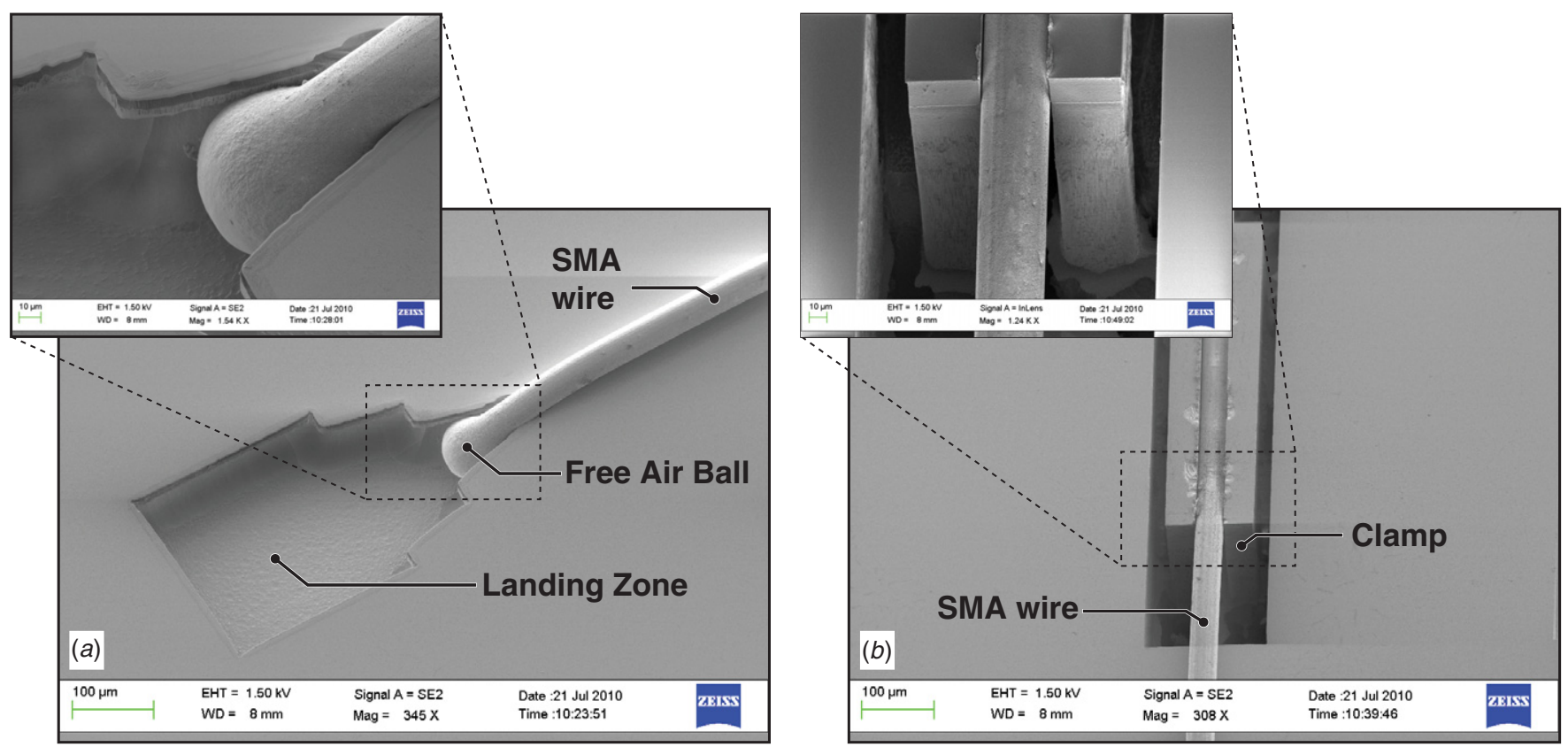

Figure 9. (a) The SEM image of the anchor structure with an anchored SMA wire. The inset shows a magnified view on the tapered design of the anchor. (b) The SEM image of a clamping structure with a clamped SMA wire. The inset is a tilted view on the deep-etched cantilevers of the clamp structures.

figure $8(b)$. Then, the SMA FAB is attached at the desired position of the anchor structure with a tapered surface opening, as depicted in figures $8(c)$ and $9(a)$. The bond capillary is then moved to the clamping structure where the bond capillary is centered and the wire is pressed between the cantilevers of the clamping structure (figures $8(d)$ and $(e)$ ). Figure $9(b)$ shows a clamped SMA wire. On the surface of the clamp structures, four imprints from the bond capillary were observed. No visible damage or deformation of the SMA wire occurs. The inset in figure $9(b)$ depicts that the SMA wire is correctly fixated in the notch on the upper end of the cantilevers.

As illustrated in figures $8(f)$ and $(g)$, the bond capillary is moved behind the clamping structure and the SMA wire is truncated with the maximum bond force of $2 \mathrm{~N}$ and a high ultrasonic energy of $2.5 \mathrm{~W}$ at $63 \mathrm{kHz}$. Figure 10 depicts a truncated SMA wire. A distinct, circular imprint from the bond capillary remains on the silicon surface after the truncation of the SMA wire. A clear cutting line without any visible deformation of the SMA wire indicates the extreme hardness of the NiTi material. Also due to the hardness of the wire and the applied bond forces, an increased wear of the bond capillary is likely; hence, the lifetime of the capillary is expected to be considerable reduced. Clogging of the bond capillary that is

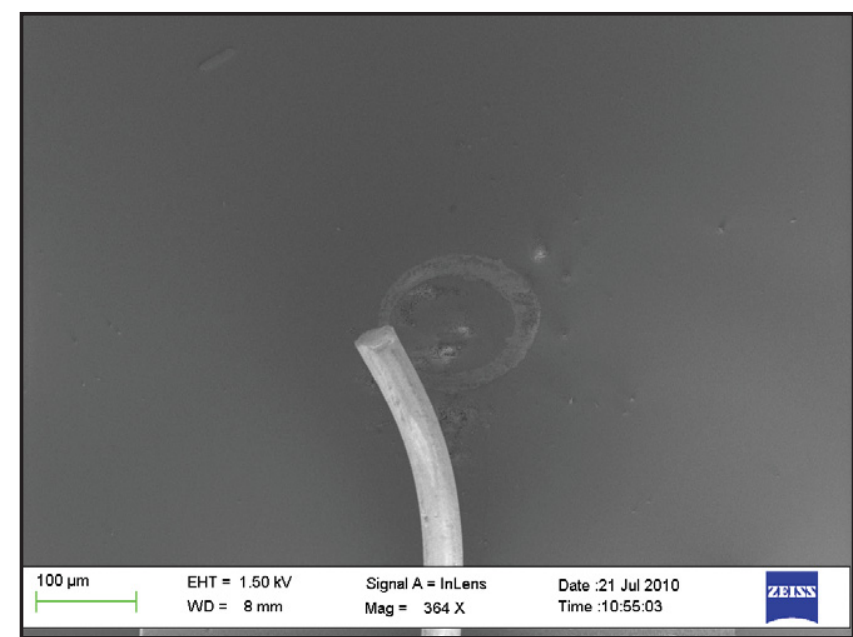

Figure 10. A truncated SMA wire and an imprint from the bond capillary on the silicon surface. The SMA wire was truncated with the maximum bond force and ultrasonic energy, which did not lead to a visible deformation of the wire.

common for soft wire materials and high bond forces did not occur during all experiments with SMA wires. 
Table 4. Results of destructive wire pull tests for SMA wires that have been fixated in anchor and clamp structures. The ultimate strength of a plain SMA wire serves as a reference.

\begin{tabular}{lllll}
\hline Experiment & Structure & Feature & Load $[\mathrm{mN}]$ & Failure mode \\
\hline Reference & SMA wire & Diameter: $37.5 \mu \mathrm{m}$ & $1374 \pm 0.5 \%$ & SMA wire failed \\
1 & Anchor & Tapered, figure 4 $(a)$ & $1241 \pm 0.5 \%$ & SMA wire failed \\
2 & Anchor & Tapered, figure 4(a) & $1350 \pm 0.5 \%$ & SMA wire failed \\
3 & Anchor & Tapered, figure 4(a) & $1313 \pm 0.5 \%$ & SMA wire failed \\
4 & Anchor & Tapered, figure 4(a) & $1301 \pm 0.5 \%$ & SMA wire failed \\
5 & Anchor & Tapered, figure 4(a) & $1318 \pm 0.5 \%$ & SMA wire failed \\
6 & Clamps & $24 \mu \mathrm{m}$ gap width & $1386 \pm 0.5 \%$ & SMA wire failed \\
7 & Clamps & $27 \mu \mathrm{m}$ gap width & $1046 \pm 0.5 \%$ & SMA wire pulled out \\
8 & Clamps & $30 \mu \mathrm{m}$ gap width & $764 \pm 0.5 \%$ & SMA wire pulled out \\
\hline
\end{tabular}

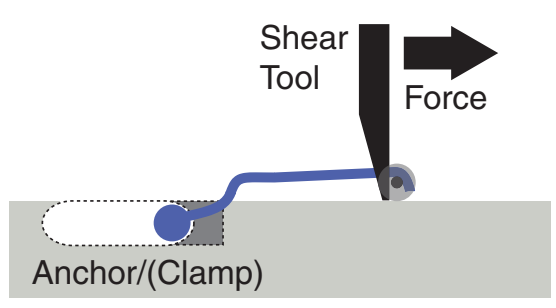

Figure 11. One end of an SMA wire which was fixated either by an anchor or a clamp structure. The other end was fixated by the shear testing cartridge, which pulled the wire laterally and thereby simulated the forces during an actual actuation.

\section{Characterization and results}

For applications in which the wires are used as an actuator, it is important to ensure a mechanically stable and reliable attachment of the wire to the substrate. Therefore, the integration method was characterized in terms of mechanical stability as well as placement accuracy of the SMA wires. A Dage PC2400 shear/pull tester was used to characterize the mechanical strength of the attachment of the wire to the anchor and clamp structures. As shown in figure 11, one end of an SMA wire with a diameter of $37.5 \mu \mathrm{m}$ was fixated either by an anchor or a clamp structure. The other end was fixated by the shear testing cartridge, which pulled the wire laterally and thereby simulated the forces during an actual actuation. All pull tests were performed with a constant pull speed of $25 \mathrm{~mm} \mathrm{~s}^{-1}$. The measured ultimate strength of the SMA wire with a diameter of $37.5 \mu \mathrm{m}$ is $1374 \mathrm{mN}$ and serves as a reference, as shown in table 4 . Wires that were fixated by an anchor structure broke consistently in the region close to the FAB. The applicable load was on the order of the ultimate strength of the SMA wire, which indicated both a reliable $\mathrm{FAB} /$ wire interface and a sufficient mechanical strength of the anchor structure. The anchoring of the FAB in the anchor structures with tapered surface openings (figure 12(b)) has a superior performance compared to anchor structures with straight surface openings (figure 12(a)). The tapered design centers the FAB and the wire and is forgiving to non-centered or non-spherical FABs. Tapered surface openings can also be used for wires and FABs with different diameters. Figure 12(b) shows a poorly anchored SMA FAB in an anchor structure with straight surface opening. The FAB imposes high stresses on the anchor structure, which forces parts of the under-etched silicon upward on the right side. In extreme cases, this can lead to a fracture of the silicon structures.

Pull tests to evaluate the mechanical strength of the attachment of the wire to the clamping structures have been performed as well. A direct dependence of the width of the
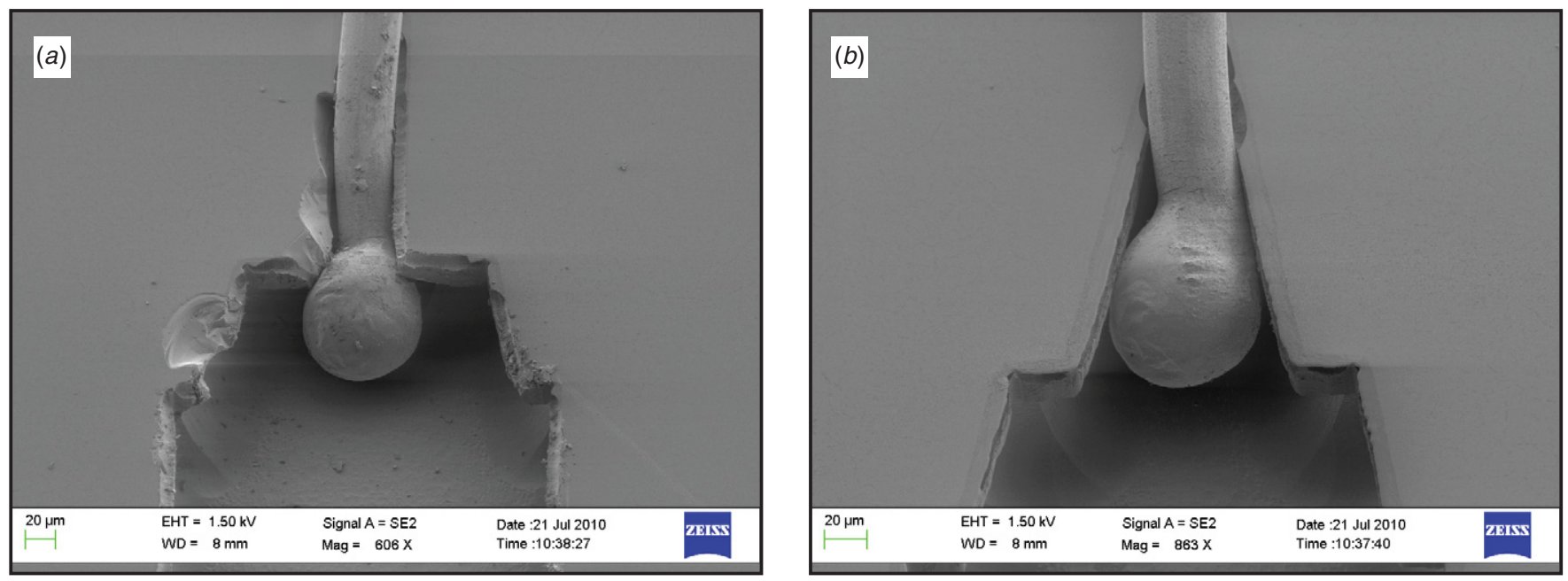

Figure 12. SEM images of the anchoring of deformed FABs. (a) The integration of a deformed FAB in an anchor with a rectangular design causes increased stresses, which can crack and destroy the silicon structure. $(b)$ The integration of a deformed FAB in an anchor with a tapered design is more reliable and does not harm the silicon structure. 
(a)

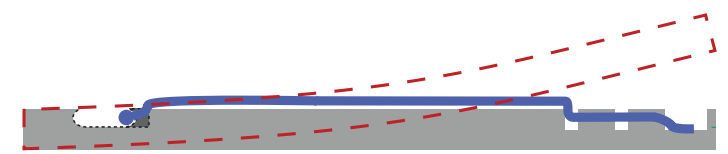

(b)

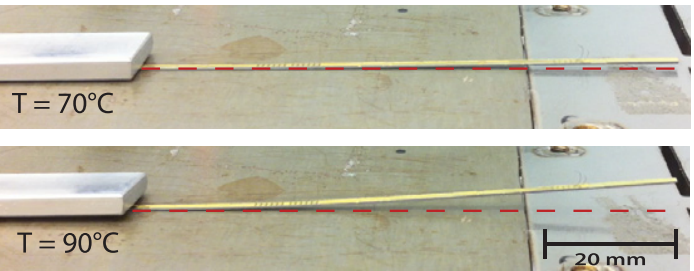

Figure 13. In order to verify the mechanical stability of the presented wire fixation approach, two $75 \mathrm{~mm}$ long SMA wires have been integrated in parallel on a silicon chip with a size of $2 \mathrm{~mm} \times$ $100 \mathrm{~mm}$. (a) Drawing of a cross-section, the red dashed outline represents the chip in a hot state. (b) Image of a slightly actuated device on a hotplate with a temperature of $70{ }^{\circ} \mathrm{C}$. $(c)$ Device with increased actuation at $90{ }^{\circ} \mathrm{C}$.

gap between the facing cantilevers on the mechanical strength of the clamping structure has been observed. Clamps with a gap width of $24 \mu \mathrm{m}$ support a sufficiently high clamping force, whereas the wire could be pulled out of clamps with gap widths greater than $27 \mu \mathrm{m}$. This is expected as the smaller the gap width of a clamping structure, the higher the spring force of the clamp cantilevers. A higher spring force ultimately results in a stronger fixation of the wire by the clamp. However, there is a lower limit of the gap distance, which is defined by the mechanical properties of silicon and the maximum applicable bond force, which the tool can apply in order to press the wire into the clamp.

For most applications, it is required that the pull force for a wire with a diameter of $37.5 \mu \mathrm{m}$ must not exceed a pull force of $200 \mathrm{mN}$ in order to prevent permanent deformation and degradation of the SMA material [29]. All measured fixation strengths of the clamping and anchoring structures are well above the maximum allowed pull force for the wire. Therefore, the presented fixation structures enable the operation of SMA wires within their specifications for actuator applications.

A simple SMA actuator was implemented in order to demonstrate the mechanical robustness of the anchor and clamping structures. This actuator is based on a $300 \mu \mathrm{m}$ thick silicon cantilever beam with a length of $100 \mathrm{~mm}$ and a width of $2 \mathrm{~mm}$ as cold state reset spring and two parallel integrated $75 \mathrm{~mm}$ long SMA wires with a diameter of $37.5 \mu \mathrm{m}$ as actuators. The energy input is provided indirectly by a hotplate on which the actuator is fixed on one side to enhance the thermal contact. Figure 13(a) illustrates the cross-section of the actuator both in actuated and idle positions. Figure 13(b) and $(c)$ depict the actuator in a semi- and a fully-actuated state, respectively. This demonstrates that both SMA wire fixation elements withstand the forces that are generated by the SMA wires.

A basic electrical measurement was performed in order to investigate the capability of the anchoring and clamping structures to create an electrical contact to the fixated wire. For this experiment, a TiW/Au film $(50 / 500 \mathrm{~nm})$ was sputter deposited on both fixation structures prior to the integration of an SMA wire with a length of $2.5 \mathrm{~cm}$. The measurement of the

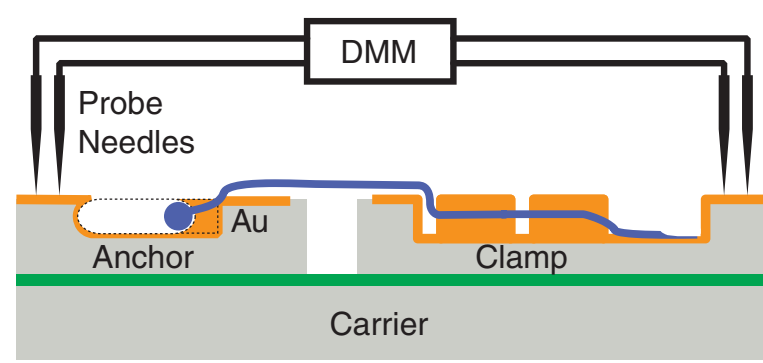

Figure 14. The setup for the resistance measurement: the probe needles were placed on the Au-coated substrate surface in close proximity to the anchor and clamp structures.
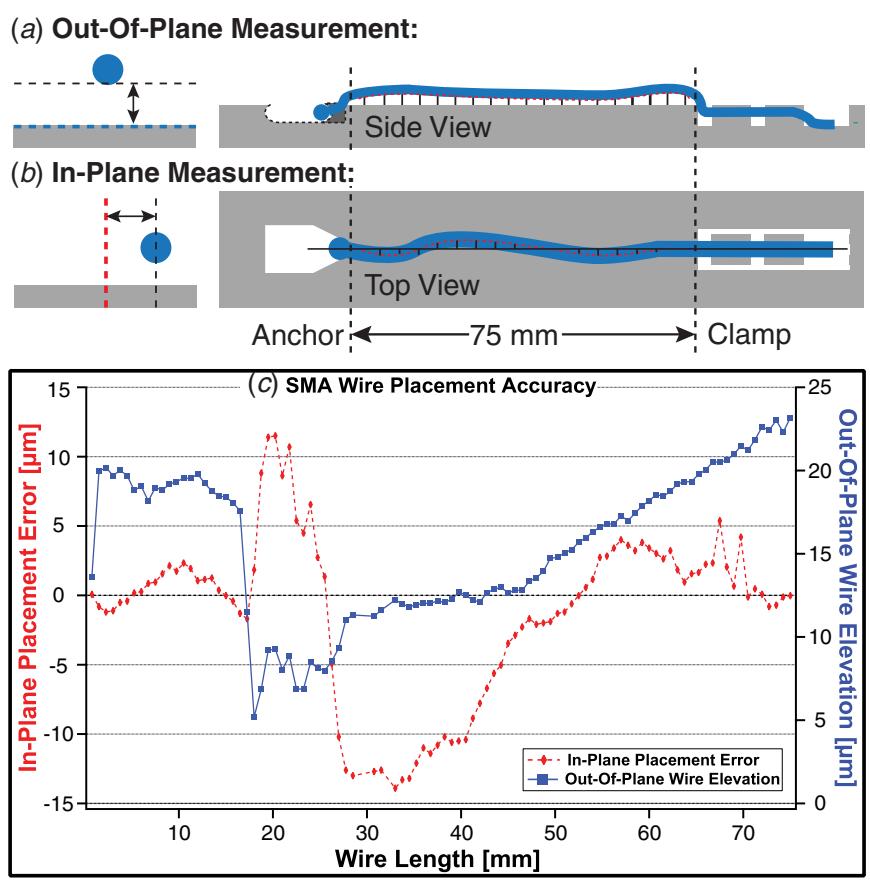

Figure 15. White light interferometric measurement data for the alignment accuracy of an SMA wire with a length of $75 \mathrm{~mm}$ and a diameter of $37.5 \mu \mathrm{m}$. As indicated in the drawing, the out-of-plane measurement (straight graph) determines the distance between the wire and the substrate, the in-plane measurement (dashed graph) determines the lateral wire placement deviation from a geometrical anchor-to-clamp center line.

resistance was performed with a four-point probe station and a digital multimeter. The measured total resistance is a series resistance that consists of the resistance of the SMA wire, the contact resistance of the SMA wire to both fixation structures and the resistance of the Au metallization. As depicted in figure 14, the probe needles were placed on the Au-coated substrate surface in close proximity to the anchor and clamp structures. The measured total resistance was less than $28 \Omega$. The contact resistance of both fixation structures and the resistance of the Au metallization was determined to approximately $6 \Omega$ by subtracting the theoretical wire resistance of $22 \Omega$ [29]. This demonstrates that the fixation structures can both mechanically fixate and contact the wire electrically and hence enables the fabrication of direct jouleheated actuator devices.

The accuracy of the wire placement has been evaluated, since it can have an impact on the design of an actual device 
utilizing this integration method. Therefore, one SMA wire with a diameter of $37.5 \mu \mathrm{m}$ was integrated on a $100 \mathrm{~mm}$ silicon substrate. The anchor-to-clamp distance (i.e. wire length) was $75 \mathrm{~mm}$. An optical profilometer (Wyko NT9300) was used to determine both the in-plane and the out-of-plane placement accuracy of the wire. One measurement consisting of 100 equidistant measurement points with a pitch of $0.75 \mathrm{~mm}$ along the ideal geometrical anchor-to-clamp center line has been performed. Each measurement on each point gives both the out-of-plane distance of the wire to the substrate (figure 15(a)) and the in-plane deviation of the position of the wire from the ideal geometrical center line (figure 15(b)). The out-of-plane measurement was determined to $15.6 \mu \mathrm{m}$ in average, and the maximum stand-off was $23.2 \mu \mathrm{m}$. This measurement revealed that the integrated wire is, apart from the fixation structures, not in contact with the substrate surface. As depicted in figure $15(c)$, the elevation of the wire is slightly higher close to the fixation structures and lower in between the fixation structures. The in-plane measurement provided information about the achievable alignment precision of the wire. The in-plane deviation from the ideal center line was determined to $4.2 \mu \mathrm{m}$ in average, the maximum deviation was $13.9 \mu \mathrm{m}$. These accuracies are sufficient for most MEMS applications; however, an automated placement with high accuracy with the help of an automated wire-bonding tool would potentially increase the quality of the wire loop.

\section{Conclusions}

A proof-of-concept of the wire-bonder-assisted integration of NiTi SMA wires to $\mathrm{Si}$ substrates was successfully demonstrated. Even though the wire has been integrated with the help of a manual wire bonder, an excellent placement accuracy could be achieved. The in-plane placement accuracy of the wires is within $14 \mu \mathrm{m}$ over a length of $75 \mathrm{~mm}$ and the mechanical fixation strength is higher than the ultimate strength of the NiTi wire.

This wire-integration approach is generic and has the potential to be applied not only to SMAs for actuator applications such as microvalves but also to other nonbondable wire materials such as steel or titanium wires, and even optical fibers. The presented manual integration approach can potentially be implemented on fully automated wirebonding tools in order to increase reliability and throughput. With highly efficient conventional wire-bonding tools broadly available, this novel mechanical fixation approach lowers the barriers for a wider use of unconventional wire materials in MEMS and other microsystems.

\section{Acknowledgments}

This work has been funded by the European Research Council (ERC) through the Advanced Grant (267528) and the Starting Grant (277879).

\section{References}

[1] Greig W J 2007 Integrated Circuit Packaging, Assembly and Interconnections (Berlin: Springer)
[2] Harman G G 2010 Wire Bonding in Microelectronics: Materials, Processes, Reliability, and Yield 3rd edn (New York: McGraw-Hill)

[3] Prasad S K 2004 Advanced Wirebond Interconnection Technology 1st edn (Dordrecht: Kluwer)

[4] Bouendeu E, Greiner A, Smith P and Korvink J 2011 A low-cost electromagnetic generator for vibration energy harvesting IEEE Sensors J. 11 107-13

[5] Badilita V, Kratt K, Baxan N, Anders J, Elverfeldt D, Boero G, Hennig J, Korvink J and Wallrabe U 2011 3D solenoidal microcoil arrays with CMOS integrated amplifiers for parallel MR imaging and spectroscopy IEEE 24th Int. Conf. Micro Electro Mechanical Systems (MEMS) pp 809-12

[6] Cho E, Lee S, Lee J and Nam S 2010 A high-efficient transformer using bond wires for Si RF IC IEICE Trans. Electron. 93 140-1

[7] Willmot R, Kim D and Peroulis D 2009 A Yagi-Uda array of high-efficiency wire-bond antennas for on-chip radio applications IEEE Trans. Microw. Theory Tech. 57 3315-21

[8] Jackson N and Muthuswamy J 2009 Flexible chip-scale package and interconnect for implantable mems movable microelectrodes for the brain J. Microelectromech. Syst. 18 396-404

[9] Baron J 2010 Stud bumping serves as TSV alternative for BSI image sensor in latest iphone 4 Yole Development, Technical Report 17

[10] Fischer A, Grange M, Roxhed N, Weerasekera R, Pamunuwa D, Stemme G and Niklaus F 2011 Wire-bonded through-silicon vias with low capacitive substrate coupling J. Micromech. Microeng. 21085035

[11] Antelius M, Fischer A, Niklaus F, Stemme G and Roxhed N 2011 Hermetic integration of liquids in mems by room temperature, high-speed plugging of liquid-filled cavities at wafer level IEEE 24th Int. Conf. Micro Electro Mechanical Systems (MEMS) pp 356-9

[12] Thiede H P 1982 Bonding wire today and tomorrow IEEE 2nd Int. Conf. Electronics Packaging (IEPS) pp 686-705

[13] Bischoff A, Aldinger F and Heraeus W 1984 Reliability criteria of new low cost materials for bonding wires and substrates 34th Conf. on IEEE Electronic Components pp 411-7

[14] Mantese J and Alcini W 1988 Platinum wire wedge bonding: a new IC and microsensor interconnect J. Electron. Mater. 17 285-9

[15] Burla R, Chen L, Zorman C and Mehregany M 2009 Development of nickel wire bonding for high-temperature packaging of SiC devices IEEE Trans. Adv. Packag. 32 564-74

[16] Heuck N N and Heuck N 2010 Development of a wire-bond technology for $\mathrm{SiC}$ high temperature applications Mater. Sci. Forum 645749

[17] Hirshberg A and Elata D 2008 Direct wire bonding to silicon devices without the use of metallic layers ASME Conf. Proc. pp 303-6

[18] Material property database, available at http://www.matweb.com/

[19] Gall K, Juntunen K, Maier H, Sehitoglu H and Chumlyakov Y 2001 Instrumented micro-indentation of NiTi shape-memory alloys Acta Mater. 49 3205-17

[20] Krulevitch P, Lee A P, Ramsey P B, Trevino J C, Hamilton J and Northp M A 1996 Thin film shape memory alloy microactuators J. Microelectromech. Syst. 5 270-82

[21] Mineta T and Haga Y 2011 Materials and processes in shape memory alloy MEMS Materials and Processes Handbook (MEMS Reference Shelf) ed R Ghodssi and P Lin (New York: Springer) pp 355-402

[22] Kohl M 2004 Shape Memory Microactuators (Berlin: Springer) 
[23] Lapisa M, Stemme G and Niklaus F 2011 Wafer-level heterogeneous integration for MOEMS, MEMS and NEMS IEEE J. Sel. Top. Quantum Electron. 17 629-44

[24] Braun S, Sandstrom N, Stemme G and van der Wijngaart W 2009 Wafer-scale manufacturing of bulk

shape-memory-alloy microactuators based on adhesive bonding of titanium nickel sheets to structured silicon wafers J. Microelectromech. Syst. 18 1309-17

[25] Gradin H, Braun S, Stemme G and van der Wijngaart W 2011 SMA microvalves for very large gas flow control manufactured using wafer-level eutectic bonding IEEE Trans. Ind. Electron.

[26] Clausi D, Gradin H, Braun S, Peirs J, Stemme G, Reynaerts D and van der Wijngaart W 2010 Design and wafer-level fabrication of SMA wire microactuators on silicon J. Microelectromech. Syst. 19 982-91

[27] Clausi D, Gradin H, Braun S, Peirs J, Reynaerts D, Stemme G and van der Wijngaart W 2011 Wafer-level mechanical and electrical integration of SMA wires to silicon MEMS using electroplating IEEE 24th Int. Conf. Micro Electro Mechanical Systems (MEMS) pp 1281-4
[28] Fischer A, Gradin H, Braun S, Schroder S, Stemme G and Niklaus F 2011 Wafer-level integration of niti shape memory alloy wires for the fabrication of microactuators using standard wire bonding technology IEEE 24th Int. Conf. Micro Electro Mechanical Systems (MEMS) pp 348-51

[29] Technical characteristics of flexinol actuator wire, available at http://www.dynalloy.com/pdfs/TCF1140.pdf

[30] Zhang W, Zhang W, Turner K and Hartwell P G 2004 Scream'03: a single mask process for high- $q$ single crystal silicon MEMS ASME Conf. Proc. pp 211-5

[31] Kratt K, Badilita V, Burger T, Mohr J, Börner M, Korvink J and Wallrabe U 2009 High aspect ratio PMMA posts and characterization method for micro coils manufactured with an automatic wire bonder Sensors Actuators A $156328-33$

[32] Duerig T 1990 Engineering Aspects of Shape Memory Alloys (England: Butterworth-Heinemann)

[33] Toyozawa K, Fujita K, Minamide S and Maeda T 1990 Development of copper wire bonding application technology IEEE Trans. Compon., Packag., Manuf. Technol. $13667-72$ 